\title{
A NOTE ON THE MINIMAL ESSENTIAL SET OF COINCIDENT POINTS FOR SET-VALUED MAPPINGS
}

\author{
LUO QUN
}

Received 12 May 2004 and in revised form 23 November 2004

Motivated by the ideas of Kinoshita, we introduce the concept of minimal essential set of the coincident points for set-valued mappings, and we prove that there exists at least one minimal essential set and one essential component of the coincident points for set-valued mappings (satisfying some conditions).

\section{Introduction}

Kinoshita [3] introduced the notion of essential component to the set of fixed points and proved that for any continuous mapping of the Hilbert cube into itself, there exists at least one essential component of the set of its fixed points. The natural extension of fixed point theory is the study of coincident points. Tan et al. [5] introduced the concept of essential coincident points for multivalued mappings, they also discussed the generic stability of coincident points for multivalued mappings. However, as can be seen in Example 2.9, there exist no essential coincident points.

In this paper, motivated by the ideas of Kinoshita, we introduce the concept of minimal essential set of the coincident points for set-valued mappings, and we prove that there exists at least one minimal essential set of the coincident points for set-valued mappings (satisfying some conditions), and hence there exists at least one essential component of the coincident points.

\section{Preliminaries}

Let $K$ be a subset of a metric space $(E, d)$; for any $\delta>0$, we denote by $O(K, \delta)=\{x \in E$ : $d(x, K)<\delta\}$ the open neighborhood of $K$ with radius $\delta$ in $E$.

Let $X$ be a nonempty compact convex subset of a Banach space $V$. Let

$$
S=\left\{f: X \longrightarrow 2^{X} \text { upper semicontinuous and nonempty closed convex values }\right\},
$$

where $2^{X}$ denotes the family of all nonempty subsets of $X$.

For any $f, f^{\prime} \in S$, define

$$
\rho_{1}\left(f, f^{\prime}\right)=\sup _{x \in X} H\left(f(x), f^{\prime}(x)\right),
$$


where $H$ is the Hausdorff metric defined on $X$. Clearly, $\left(S, \rho_{1}\right)$ is a complete metric space. Let

$$
Y=\left\{(f, g) \in S \times S: f, g \in S, \text { for any } x \in \operatorname{Bd} X,(f(x)-g(x)) \cap\left(\bigcup_{\lambda>0} \lambda(X-x)\right) \neq \varnothing\right\},
$$

where $\mathrm{Bd} X$ denotes the boundary of $X$, then $(Y, \rho)$ is a complete metric space, where $\rho\left((f, g),\left(f^{\prime}, g^{\prime}\right)\right)=\rho_{1}\left(f, f^{\prime}\right)+\rho_{1}\left(g, g^{\prime}\right)$.

Theorem 2.1. $Y \subset S \times S$ is a closed subset.

Proof. Let $y_{\alpha}=\left(f_{\alpha}, g_{\alpha}\right) \in Y$ with $y_{\alpha} \rightarrow y=(f, g) \in S \times S$. Since $\left(f_{\alpha}, g_{\alpha}\right) \in Y$, for any $x \in$ $\operatorname{Bd} X$, one has

$$
\left(f_{\alpha}(x)-g_{\alpha}(x)\right) \cap\left(\bigcup_{\lambda>0} \lambda(X-x)\right) \neq \varnothing
$$

Then there exist $u_{\alpha} \in f_{\alpha}(x)$ and $v_{\alpha} \in g_{\alpha}(x)$ such that

$$
u_{\alpha}-v_{\alpha} \in \bigcup_{\lambda>0} \lambda(X-x)
$$

Note that $f_{\alpha} \rightarrow f, g_{\alpha} \rightarrow g, X$ is compact, $\left\{u_{\alpha}\right\}$ has a cluster point $u_{0} \in f(x)$, and $\left\{v_{\alpha}\right\}$ has a cluster point $v_{0} \in g(x)$. Without loss of generality, we may assume that $u_{\alpha} \rightarrow u_{0} \in f(x)$, $v_{\alpha} \rightarrow v_{0} \in g(x)$.

(1) If there exists infinite $\alpha$ such that $u_{\alpha}=v_{\alpha}$, then $u_{0}=v_{0}$, and hence $u_{0}-v_{0} \in$ $\bigcup_{\lambda>0} \lambda(X-x)$.

(2) If there exists infinite $\alpha$ such that $u_{\alpha} \neq v_{\alpha}$, then there exists $k>0$ such that

$$
u_{\alpha}-v_{\alpha} \in \bigcup_{0<\lambda<k} \lambda(X-x)
$$

Hence there exists $\lambda_{\alpha}$ with $0<\lambda_{\alpha}<k$ such that $u_{\alpha}-v_{\alpha} \in \lambda_{\alpha}(X-x)$. So there exists $z_{\alpha} \in X$ such that $u_{\alpha}-v_{\alpha}=\lambda_{\alpha}\left(z_{\alpha}-x\right)$. Note that $X$ is compact, $\left\{z_{\alpha}\right\}$ has a cluster point $z_{0} \in X$, we may assume that $z_{\alpha} \rightarrow z_{0}$. And since $0<\lambda_{\alpha}<k$, we may assume that $\lambda_{\alpha} \rightarrow \lambda_{0}(\geq 0)$, hence

$$
u_{0}-v_{0}=\lambda_{0}\left(z_{0}-x\right) .
$$

If $\lambda_{0}=0$, then $u_{0}-v_{0}=0 \in \bigcup_{\lambda>0} \lambda(X-x)$. If $\lambda_{0} \neq 0$, then $u_{0}-v_{0}=\lambda_{0}\left(z_{0}-x\right) \in$ $\lambda_{0}(X-x) \subset \bigcup_{\lambda>0} \lambda(X-x)$. Hence, for any $x \in \operatorname{Bd} X$,

$$
(f(x)-g(x)) \cap\left(\bigcup_{\lambda>0} \lambda(X-x)\right) \neq \varnothing .
$$

Therefore $Y \subset S \times S$ is a closed subset.

For any $y=(f, g) \in Y$, we denote by $C C(y)=\{x \in X: f(x) \cap g(x) \neq \varnothing\}$ the set of coincident points of the set-valued mappings $f$ and $g$, by [2, Theorem 10], $C C(y) \neq \varnothing$, thus $y \rightarrow C C(y)$ indeed defines a set-valued mapping of coincident points from $Y$ to $X$ and we have the following theorem. 
Theorem 2.2. The mapping CC: $Y \rightarrow 2^{X}$ is upper semicontinuous with nonempty compact values.

Proof. For any $y=(f, g) \in Y$, we need to prove that $C C(y) \subset X$ is compact. Let a sequence $\left\{x_{\alpha}\right\} \subset C C(y)$ and $x_{\alpha} \rightarrow x_{0} \in X$. Since $x_{\alpha} \in C C(y)$, we have $f\left(x_{\alpha}\right) \cap g\left(x_{\alpha}\right) \neq \varnothing$.

Suppose that $f\left(x_{0}\right) \cap g\left(x_{0}\right)=\varnothing$, then there exists $\delta>0$ such that

$$
O\left(f\left(x_{0}\right), \delta\right) \cap O\left(g\left(x_{0}\right), \delta\right)=\varnothing .
$$

By upper semicontinuities of $f$ and $g$, and since $x_{\alpha} \rightarrow x_{0}$, there exists $\alpha_{0}$ such that for any $\alpha>\alpha_{0}, f\left(x_{\alpha}\right) \subset O\left(f\left(x_{0}\right), \delta\right)$ and $g\left(x_{\alpha}\right) \subset O\left(f\left(x_{0}\right), \delta\right)$, then $f\left(x_{\alpha}\right) \cap g\left(x_{\alpha}\right)=\varnothing$, which contradicts the fact that $f\left(x_{\alpha}\right) \cap g\left(x_{\alpha}\right) \neq \varnothing$, hence $x_{0} \in C C(y)$ and hence $C C(y)$ is compact.

Since $X$ is compact, we want to prove that the mapping $C C$ is upper semicontinuous, we only need to prove that the Graph $C C$ of $C C$ is closed:

$$
\text { Graph } C C=\{(y, x) \in Y \times X: x \in C C(y), y \in Y\}
$$

Let a sequence $\left\{\left(y_{\alpha}, x_{\alpha}\right)\right\} \subset$ Graph $C C$ and $\left(y_{\alpha}, x_{\alpha}\right) \rightarrow\left(y_{0}, x_{0}\right) \in Y \times X$. Denote $y_{\alpha}=\left(f_{\alpha}, g_{\alpha}\right)$, $y_{0}=\left(f_{0}, g_{0}\right)$, then $x_{\alpha} \in C C\left(y_{\alpha}\right)$ and $f_{\alpha}\left(x_{\alpha}\right) \cap g_{\alpha}\left(x_{\alpha}\right) \neq \varnothing$.

Suppose that $f_{0}\left(x_{0}\right) \cap g_{0}\left(x_{0}\right)=\varnothing$, then there exists $\delta^{*}>0$ such that

$$
O\left(f_{0}\left(x_{0}\right), \delta^{*}\right) \cap O\left(g_{0}\left(x_{0}\right), \delta^{*}\right)=\varnothing .
$$

Since $f_{\alpha} \rightarrow f_{0}, g_{\alpha} \rightarrow g_{0}, x_{\alpha} \rightarrow x_{0}$, and $f_{0}, g_{0}$ are upper semicontinuous, there exists $\alpha^{*}$ such that

$$
\begin{array}{ll}
f_{\alpha}\left(x_{\alpha}\right) \subset O\left(f_{0}\left(x_{\alpha}\right), \frac{\delta^{*}}{2}\right) \subset O\left(f_{0}\left(x_{0}\right), \delta^{*}\right), & \forall \alpha>\alpha^{*}, \\
g_{\alpha}\left(x_{\alpha}\right) \subset O\left(g_{0}\left(x_{\alpha}\right), \frac{\delta^{*}}{2}\right) \subset O\left(g_{0}\left(x_{0}\right), \delta^{*}\right), \quad \forall \alpha>\alpha^{*} .
\end{array}
$$

Hence $f_{\alpha}\left(x_{\alpha}\right) \cap g_{\alpha}\left(x_{\alpha}\right)=\varnothing$, which contradicts the fact that $f_{\alpha}\left(x_{\alpha}\right) \cap g_{\alpha}\left(x_{\alpha}\right) \neq \varnothing$. So the mapping $C C$ is upper semicontinuous with nonempty compact values.

For each $y \in Y$, the component of a point $x \in C C(y)$ is the union of all connected subsets of $C C(y)$ which contain the point $x$, see [1, page 356], components are connected closed subsets of $C C(y)$ and are also connected compact. It is easy to see that the components of two distinct points of $C C(y)$ either coincide or are disjoint, so that all components constitute a decomposition of $C C(y)$ into connected pairwise disjoint compact subsets, that is,

$$
C C(y)=\bigcup_{\alpha \in \Lambda} C_{\alpha}(y)
$$

where $\Lambda$ is an index set, for any $\alpha \in \Lambda, C_{\alpha}(y)$ is a nonempty connected compact subset and for any $\alpha, \beta \in \Lambda(\alpha \neq \beta), C_{\alpha}(y) \cap C_{\beta}(y)=\varnothing$. 
Definition 2.3. For $y \in Y, C C(y)=\bigcup_{\alpha \in \Lambda} C_{\alpha}(y), C_{\alpha}(y)$ is called an essential component if for each open set $O$ containing $C_{\alpha}(y)$, there exists $\delta>0$ such that for any $y^{\prime} \in Y$ with $\rho\left(y, y^{\prime}\right)<\delta, C C\left(y^{\prime}\right) \cap O \neq \varnothing$.

Definition 2.4. For $y \in Y, e(y) \subset C C(y)$ is a nonempty closed set, $e(y)$ is called an essential set of $C C(y)$ (with respect to $Y$ ) if for any open set $U$ with $U \supset e(y)$, there is $\delta>0$ such that for any $y^{\prime} \in Y$ with $\rho\left(y, y^{\prime}\right)<\delta, C C\left(y^{\prime}\right) \cap U \neq \varnothing$.

Definition 2.5. For $y \in Y, m(y) \subset C C(y)$ is an essential set, $m(y)$ is called a minimal essential set of $C C(y)$ (with respect to $Y$ ) if $m(y)$ is a minimal element of the family of essential sets of $C C(y)$ ordered by set inclusion.

Remark 2.6. If $e_{1}(y) \subset C C(y)$ is an essential set of $C C(y)$ (with respect to $Y$ ), $e_{2}(y) \subset$ $C C(y)$ is closed, and $e_{1}(y) \subset e_{2}(y)$, then $e_{2}(y)$ is also an essential set of $C C(y)$.

Remark 2.7. If $x \in C C(y)$ is an essential coincident point (see [5]) of $C C(y)$, then $\{x\}$ is an essential set of $C C(y) ; e(y) \subset C C(y)$ is an essential set and $e(y)=\{x\}$, then $x \in C C(y)$ is an essential coincident point of $C C(y)$.

Remark 2.8. If $A \subset C C(y)$ is closed, $x \in A \subset C C(y)$, and $x$ is an essential coincident point of $C C(y)$, then $A$ is an essential set and $\{x\}$ is a minimal essential set of $C C(y)$.

Example 2.9. Let $X=[0,1]$, for any $x \in X, f(x)=[0, x], g(x)=[x, 1]$, then $y=(f, g) \in Y$ and $C C(y)=\{x \in[0,1]: f(x) \cap g(x) \neq \varnothing\}=[0,1]$. But $x_{0}$ is not an essential coincident point for any $x_{0} \in C C(y)$. If $x_{0} \in(0,1)$, for all $\varepsilon>0$, take $\delta>0(\delta<\varepsilon / 2)$ such that $O\left(x_{0}, \delta\right)=\left(x_{0}-\delta, x_{0}+\delta\right) \subset[0,1]$.

Define the set-valued mappings $f^{\varepsilon}, g^{\varepsilon}: X \rightarrow 2^{X}$ by

$$
f^{\varepsilon}(x)= \begin{cases}{[0, x],} & x \in\left[0, x_{0}-\delta\right], \\ {\left[0,\left(1-\frac{\varepsilon}{2 \delta}\right) x+\frac{\varepsilon}{2 \delta}\left(x_{0}-\delta\right)\right],} & x \in\left[x_{0}-\delta, x_{0}\right], \\ {\left[0,\left(1+\frac{\varepsilon}{2 \delta}\right) x-\frac{\varepsilon}{2 \delta}\left(x_{0}+\delta\right)\right],} & x \in\left(x_{0}, x_{0}+\delta\right], \\ {[0, x],} & x \in\left(x_{0}+\delta, 1\right],\end{cases}
$$

then $y^{\varepsilon}=\left(f^{\varepsilon}, g^{\varepsilon}\right) \in Y$ and $\rho\left(y, y^{\varepsilon}\right)<\varepsilon$, but $C C\left(y^{\varepsilon}\right) \cap O\left(x_{0}, \delta\right)=\varnothing$, hence $x_{0} \in(0,1)$ is not an essential coincident point.

Similarly, if $x_{0}=1$, for all $\varepsilon: 0<\varepsilon<1 / 2$, take $\delta>0(\delta<\varepsilon / 2)$ such that $(1-\delta, 1] \subset(0,1]$.

Define the set-valued mappings $f^{\varepsilon}, g^{\varepsilon}: X \rightarrow 2^{X}$ by

$$
f^{\varepsilon}(x)= \begin{cases}{[0, x]} & \text { if } x \in[0,1-\delta], \\ {\left[0,\left(1-\frac{\varepsilon}{2 \delta}\right) x+\frac{\varepsilon}{2 \delta}(1-\delta)\right]} & \text { if } x \in(1-\delta, 1] .\end{cases}
$$

If $x_{0}=0$, for all $\varepsilon>0(<1 / 2)$, take $\delta>0(\delta<\varepsilon / 2)$ such that $[0, \delta) \subset[0,1)$. 
Define the set-valued mappings $f^{\varepsilon}, g^{\varepsilon}: X \rightarrow 2^{X}$ by

$$
\begin{gathered}
f^{\varepsilon}(x)=f(x), \\
g^{\varepsilon}(x)= \begin{cases}{\left[\left(1-\frac{\varepsilon}{2 \delta}\right) x+\frac{\varepsilon}{2}, 1\right]} & \text { if } x \in[0, \delta], \\
{[x, 1]} & \text { if } x \in(\delta, 1] .\end{cases}
\end{gathered}
$$

Hence, for any $x_{0} \in C C(y)=[0,1], x_{0}$ is not an essential coincident point.

\section{The minimal essential set of coincident points}

By Zorn lemma, we obtain the following theorem.

Theorem 3.1. For any $y \in Y$, there exists at least one minimal essential set of CC $(y)$.

Proof. By Theorem 2.2, the map CC: $Y \rightarrow 2^{X}$ is upper semicontinuous and $C C(y)$ is compact for any $y \in Y$, then $C C(y)$ is an essential set.

Let $E(y)$ denote the family of all essential sets of $C C(y)$ ordered by set inclusion. Let $\left\{e_{\alpha}(y)\right\}_{\alpha \in \Gamma}$ be a decreasing chain of $E(y)$, then $\lim e_{\alpha}(y)=\bigcap_{\alpha \in \Gamma} e_{\alpha}(y) \neq \varnothing$ and is compact. Denoting $e(y)=\lim e_{\alpha}(y)$, we need to prove that $e(y)$ is the lower bound of the chain $\left\{e_{\alpha}\right\}_{\alpha \in \Gamma}$, that is, $e(y) \in E(y)$. Since $e_{\alpha}(y)$ is compact, by [4, page 43], $H\left(e_{\alpha}(y), e(y)\right)$ $\rightarrow 0$, where $H$ is the Hausdorff metric defined on $X$, hence for any open set $O$ with $O \supset e(y)$, there is $\alpha_{1} \in \Gamma$ such that $e_{\alpha}(y) \subset O$ for any $\alpha>\alpha_{1}$. Since $e_{\alpha}(y)$ is an essential set of $C C(y)$, there exists $\delta>0$ such that $C C\left(y^{\prime}\right) \cap O \neq \varnothing$ for any $y^{\prime} \in Y$ with $\rho\left(y, y^{\prime}\right)<\delta$, then $e(y)$ is an essential set of $C C(y), e(y)$ is the lower bound of the chain $\left\{e_{\alpha}\right\}_{\alpha \in \Gamma \text {. There- }}$ fore, by Zorn lemma, $E(y)$ has a minimal element and this minimal element is a minimal essential set of $C C(y)$.

Theorem 3.2. For any $y \in Y$, the minimal essential set of CC $(y)$ is connected.

Proof. Let $m(y)$ be a minimal essential set of $C C(y)$. Suppose that $m(y)$ was not connected, then there exist two nonempty closed sets $C_{1}(y), C_{2}(y)$ and two open $U_{1}, U_{2}$ such that $C_{1}(y) \subset U_{1}, C_{2}(y) \subset U_{2}$ and $m(y)=C_{1}(y) \cup C_{2}(y), U_{1} \cap U_{2}=\varnothing$. Because $m(y)$ is a minimal essential set of $C C(y), C_{1}(y)$ and $C_{2}(y)$ are not essential sets. Since $C_{1}(y)$ and $C_{2}(y)$ are compact, there exist two open sets is $V_{1}$ and $V_{2}$ which satisfy

$$
C_{1}(y) \subset V_{1} \subset \bar{V}_{1} \subset U_{1}, \quad C_{2}(y) \subset V_{2} \subset \bar{V}_{2} \subset U_{2},
$$

where $\bar{V}_{i}$ denotes the closure of $V_{i}, i=1,2$.

For any $\delta>0$, there exist $y_{1}=\left(f_{1}, g_{1}\right), y_{2}=\left(f_{2}, g_{2}\right) \in Y$ with $\rho\left(y, y_{1}\right)<\delta, \rho\left(y, y_{2}\right)<\delta$ such that

$$
C C\left(y_{1}\right) \cap V_{1}=\varnothing, \quad C C\left(y_{2}\right) \cap V_{2}=\varnothing
$$


Define two set-valued maps $f^{*}: X \rightarrow 2^{X}$ and $g^{*}: X \rightarrow 2^{X}$ as follows:

$$
\begin{aligned}
& f^{*}(x)= \begin{cases}f_{1}(x) & \text { if } x \in \bar{V}_{1}, \\
f_{2}(x) & \text { if } x \in \bar{V}_{2}, \\
\xi(x) f_{1}(x)+\eta(x) f_{2}(x) & \text { if } x \in X \backslash \bar{V}_{1} \cup \bar{V}_{2},\end{cases} \\
& g^{*}(x)= \begin{cases}g_{1}(x) & \text { if } x \in \bar{V}_{1}, \\
g_{2}(x) & \text { if } x \in \bar{V}_{2}, \\
\xi(x) g_{1}(x)+\eta(x) g_{2}(x) & \text { if } x \in X \backslash \bar{V}_{1} \cup \bar{V}_{2},\end{cases}
\end{aligned}
$$

where

$$
\xi(x)=\frac{d\left(x, \bar{V}_{2}\right)}{d\left(x, \bar{V}_{2}\right)+d\left(x, \bar{V}_{1}\right)}, \quad \eta(x)=\frac{d\left(x, \bar{V}_{1}\right)}{d\left(x, \bar{V}_{2}\right)+d\left(x, \bar{V}_{1}\right)}
$$

It is easy to see that $y^{*}=\left(f^{*}, g^{*}\right) \in Y$, then $C C\left(y^{*}\right) \neq \varnothing$ and $C C\left(y^{*}\right) \cap\left(V_{1} \cup V_{2}\right)=\varnothing$.

Since $\rho\left(y, y^{*}\right)=\rho_{1}\left(f, f^{*}\right)+\rho_{1}\left(g, g^{*}\right)$, by [6, Lemma 3.1], we have $\rho\left(y, y^{*}\right)<\delta$, but $m(y) \subset C_{1}(y) \cup C_{2}(y) \subset V_{1} \cup V_{2}$, by Definition 2.4, $m(y)$ is not an essential set of CC $(y)$, which contradicts the fact that $m(y)$ is a minimal essential set, hence $m(y)$ is connected and the proof is complete.

By Theorems 3.1 and 3.2, we have the following corollaries.

Corollary 3.3. For any $y \in Y$, there exists at least one connected minimal essential set of $C C(y)$.

COROLlary 3.4. For any $y \in Y$, there exists at least one essential component of CC $(y)$.

Proof. For any $y \in Y$, by Corollary 3.3, there exists at least one connected minimal essential set $m(y)$ of $C C(y)$, since $m(y)$ is connected, there exists a component $M(y)$ of $C C(y)$ such that $m(y) \subset M(y)$, by Definition 2.3, M(y) is an essential component of CC $(y)$.

Remark 3.5. If $g(x)=x$ for any $x \in X$, then for any $f \in S$ and $x \in \operatorname{Bd} X$,

$$
(f(x)-g(x)) \cap\left(\bigcup_{\lambda>0} \lambda(X-x)\right)=(f(x)-x) \cap\left(\bigcup_{\lambda>0} \lambda(X-x)\right) \neq \varnothing
$$

Therefore $y=(f, g) \in Y$ and $C C(y)=F(f)$, where $F(f)$ denotes the set of fixed points of $f$.

By Corollary 3.4, we have the following corollary.

Corollary 3.6. For any $f \in S$, there is at least one essential component of $F(f)$.

Remark 3.7. Corollary 3.6 is a generalization of [3, Theorem 3]. 
Example 3.8. Let $X=[-1,1]$,

$$
\begin{aligned}
& f(x)= \begin{cases}\{0\}, & -1 \leq x<0, \\
{[0, x],} & 0 \leq x \leq 1,\end{cases} \\
& g(x)= \begin{cases}{[x,-1],} & -1 \leq x<0, \\
{[0, x-1],} & 0 \leq x \leq 1 .\end{cases}
\end{aligned}
$$

Then $y=(f, g) \in Y$ and $C C(y)=\{x \in[0,1]: f(x) \cap g(x) \neq \varnothing\}=[0,1] \subset[-1,1]$.

Suppose that $[0,1]$ is not an essential set of $C C(y)$, then there exists an open set $U$ with $U \supset[0,1]($ Let $U=(-\varepsilon, 1], 0<\varepsilon<1)$, for all $\delta>0$, there exists $y^{\delta} \in Y$ with $H\left(y, y^{\delta}\right)<\delta$ such that $C C\left(y^{\delta}\right) \cap U=\varnothing$, that is, $C C\left(y^{\delta}\right) \subset[-1,-\varepsilon]$.

Take $\delta=\varepsilon / 4$, for any $y^{0}=\left(f^{0}, g^{0}\right) \in Y$ with $\rho_{1}\left(f, f^{0}\right)<\delta / 2$ and $\rho_{1}\left(g, g^{0}\right)<\delta / 2$, one has $\rho\left(y, y^{0}\right)<\delta$, and for all $x \in[-1,-\varepsilon], H\left(f(x), f^{0}(x)\right)<\rho_{1}\left(f, f^{0}\right)<\delta / 2, H\left(g(x), g^{0}(x)\right)<$ $\rho_{1}\left(g, g^{0}\right)<\delta / 2$, then $f^{0}(x) \subset(-\delta / 2, \delta / 2), g^{0}(x) \subset[-\varepsilon+\delta / 2,-1]=[(-7 / 2) \delta,-1]$, and $[-\delta / 2, \delta / 2] \cap[(-7 / 2) \delta,-1]=\varnothing$, hence $f^{0}(x) \cap g^{0}(x)=\varnothing$ for any $x \in[-1,-\varepsilon], C C\left(y^{0}\right)$ $\subset(-\varepsilon, 1]$ which contradicts the fact that $C C\left(y^{0}\right) \subset[-1,-\varepsilon]$. Therefore, $[0,1]$ is an essential set and hence $[0,1]$ is a minimal essential set.

\section{Acknowledgment}

This research was supported by the Natural Science Foundation of Guangdong Province, China.

\section{References}

[1] R. Engelking, General Topology, Heldermann, Berlin, 1980.

[2] K. Fan, Some properties of convex sets related to fixed point theorems, Math. Ann. 266 (1984), no. 4, 519-537.

[3] S. Kinoshita, On essential components of the set of fixed points, Osaka Math. J. 4 (1952), 19-22.

[4] E. Klein and A. C. Thompson, Theory of Correspondences, Canadian Mathematical Society Series of Monographs and Advanced Texts, John Wiley \& Sons, New York, 1984.

[5] K. K. Tan, J. Yu, and X. Z. Yuan, The stability of coincident points for multivalued mappings, Nonlinear Anal. Series A: Theory and Methods 25 (1995), no. 2, 163-168.

[6] J. Yu and Q. Luo, On essential components of the solution set of generalized games, J. Math. Anal. Appl. 230 (1999), no. 2, 303-310.

Luo Qun: Department of Mathematics, Zhaoqing University, Zhaoqing, Guangdong 526061, China

E-mail address: luoqun@zqu.edu.cn 


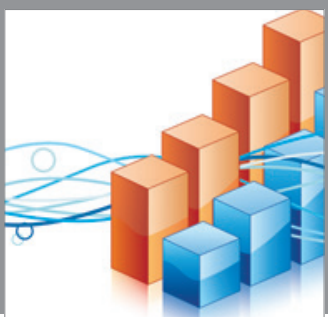

Advances in

Operations Research

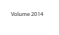

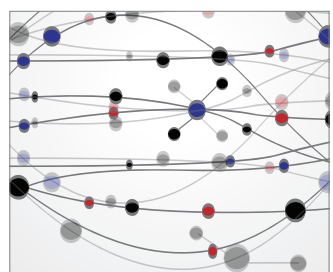

\section{The Scientific} World Journal
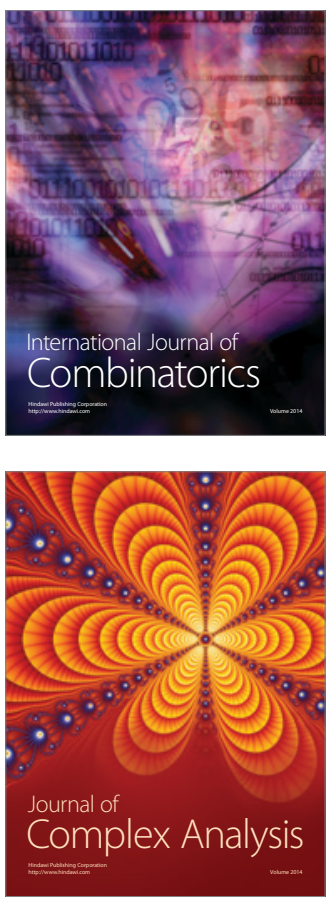

International Journal of

Mathematics and

Mathematical

Sciences
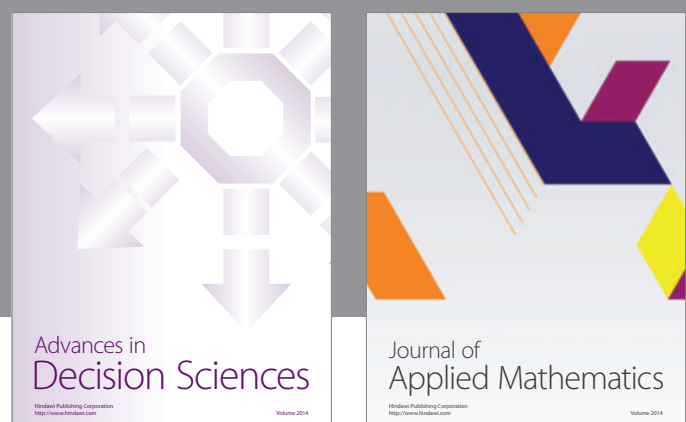

Journal of

Applied Mathematics
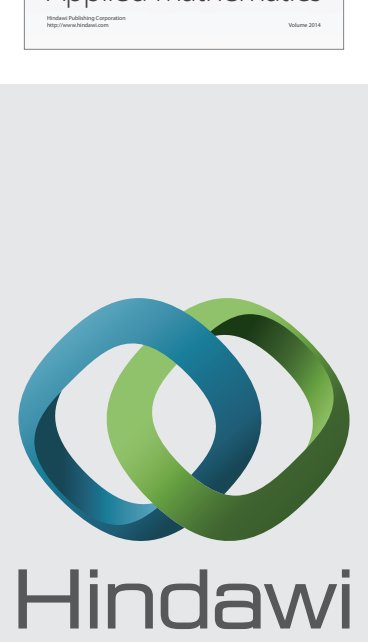

Submit your manuscripts at http://www.hindawi.com
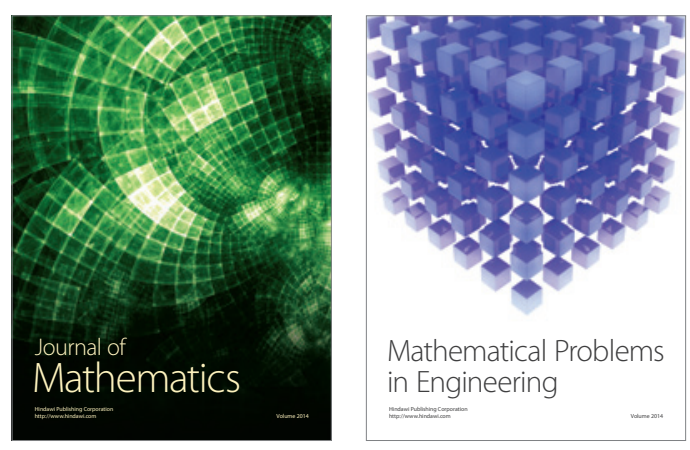

Mathematical Problems in Engineering
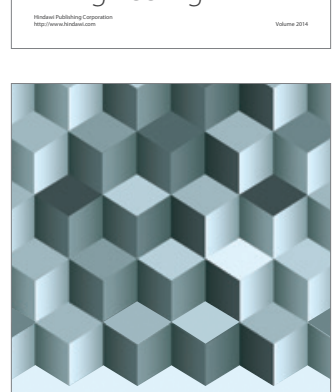

Journal of

Function Spaces
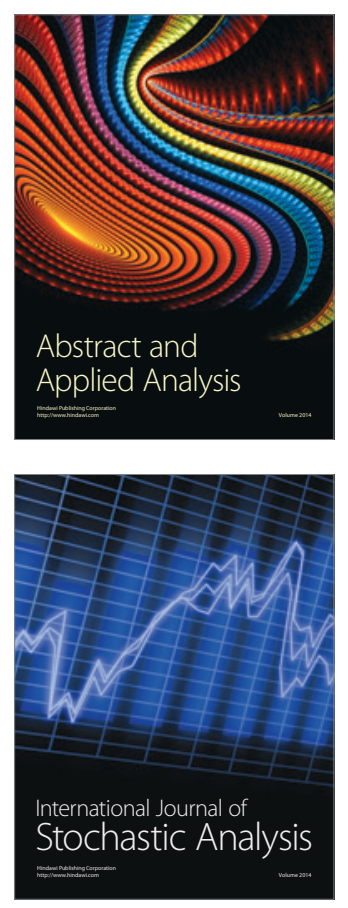

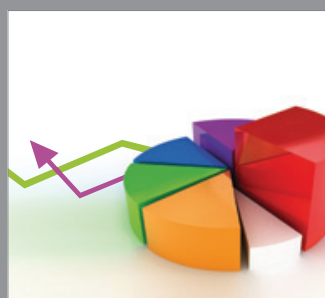

ournal of

Probability and Statistics

Promensencen
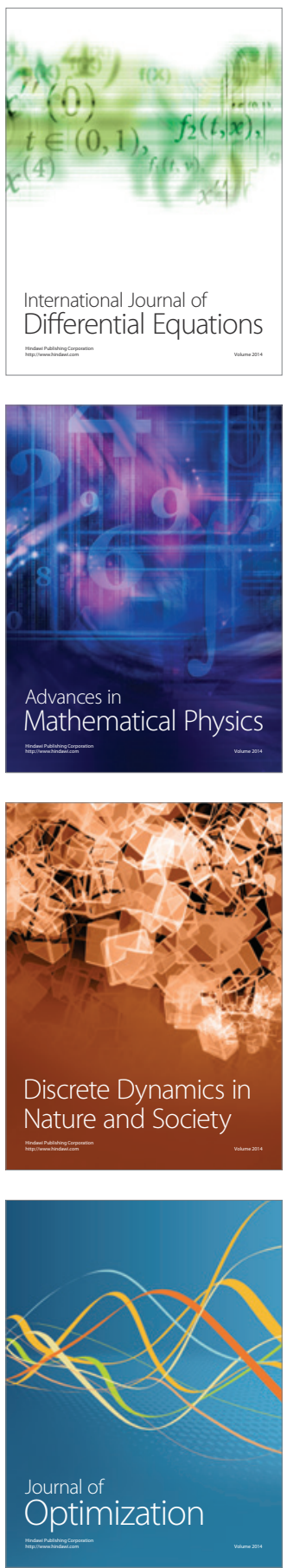